\title{
TEM studies of IniA from Mycobacterium tuberculosis
}

\author{
J. Sun*, S. Sridharan**, J. Sacchettini**, C. Savva*, A. Holzenburg*** \\ *Microscopy and Imaging Center and Dept. Biology, Texas A\&M University, College Station, \\ TX 77843-2257, USA \\ **Dept. Biochemistry and Biophysics, Texas A\&M University, College Station, TX 77843, USA
}

Mycobacterium tuberculosis is one of the main causes of death from a single infectious disease worldwide. A complete understanding of the cellular and molecular mechanisms, in particular structure-function relationships of those proteins that are intimately involved in the pathogenesis, may help design new drugs to treat this infectious bacterium. In the course of studying differential gene expression of $M$. tuberculosis, iniA, iniB and $i n i C$ genes were found to be specifically induced by a broad range of inhibitors of cell wall biosynthesis [1]. Therefore, the characterization of the proteins encoded by ini $A B C$ genes may aid in the development of new antibiotics.

The iniA gene from M. tuberculosis was expressed in E.coli with a 6-His tag and the protein purified using a Ni-column. The protein was kept in $100 \mathrm{mM}$ Tris- $\mathrm{HCl}(\mathrm{pH} 7.0), 100 \mathrm{mM} \mathrm{Li}_{2} \mathrm{SO}_{4}$ and $1 \mathrm{mM}$ DTT. Negatively stained specimens were prepared according to Valentine et al. [2] using an aqueous solution of uranyl acetate (1\% w/v, pH 4.25) and examined in a Zeiss $10 \mathrm{C}$ TEM operated at $80 \mathrm{kV}$. Micrographs were taken at a calibrated magnification $(35 \mathrm{kx})$ and digitized with a LeafScan 45 to $5.7 \AA$ / pixel at the specimen level. Particles were selected using the BOXER routine in EMAN [3], and averaged in IMAGIC V [4] after reference-free alignment, multivariate statistical analysis and automatic hierarchical classification. 3-D reconstructions were calculated and refined in EMAN.

Most projections in the electron micrographs show pronounced threefold rotational symmetry while some rectangular structures displaying two parallel lines of higher density could also be observed. The class sums after image processing suggest that IniA has trimeric features but also appears as a double-layered structure in a projection that could constitute a side-on view (Fig. 1). The overall dimensions of these particles are approximately $135 \AA$ across and $80 \AA$ in height. Each of the protein deficits present in the face-on view measures approximately $30 \AA$ in diameter. In Fig. 2, surface-rendered 3-D reconstructions are presented assuming one axis of threefold rotational symmetry and a molecular mass of $219 \mathrm{kDa}$ (top row) as well as $438 \mathrm{kDa}$ (bottom row). With the molecular mass of an IniA monomer being $73 \mathrm{kDa}$, this would mean that the structure shown is either a trimer or a hexamer. The missing densities in the $219 \mathrm{kDa}$ rendering and the fact that the $438 \mathrm{kDa}$ reconstruction better reflects the 2-D projections obtained prior to imposing symmetry suggest that IniA is a hexamer. It is interesting to note that in addition to the essentially triangular IniA, a hexameric ring form was observed (Fig. 3). These two forms could constitute different configurational states of IniA.

Furthermore, in the presence high salt concentrations (3M sodium formate, $2 \% \mathrm{w} / \mathrm{v}$ phosphotungstic acid, $\mathrm{pH}$ 6.5), small IniA crystals were obtained in which the protein appears to have assumed a tetrameric state (Fig. 4). 
$\underline{\text { References }}$

1. D. Alland, A.J. Steyn, T. Weisbrod, K. Aldrich, and W. R. Jacobs, J. Bacteriology, 182: 18021811 (2000).

2. R.C. Valentine, B.M. Shapiro and E.R. Stadtman, Biochemistry 7: 2143-2152 (1968).

3. S.J.Ludtke, J. Jakana, J.L.Song, D.T.Chuang, W.Chiu, J Mol Biol 314:253-62 (2001).

4. M.V.Heel, G.Harauz, E.Orlova, R.Schmidt, M.Schatz, J Struct Biol 116:17-24 (1996).

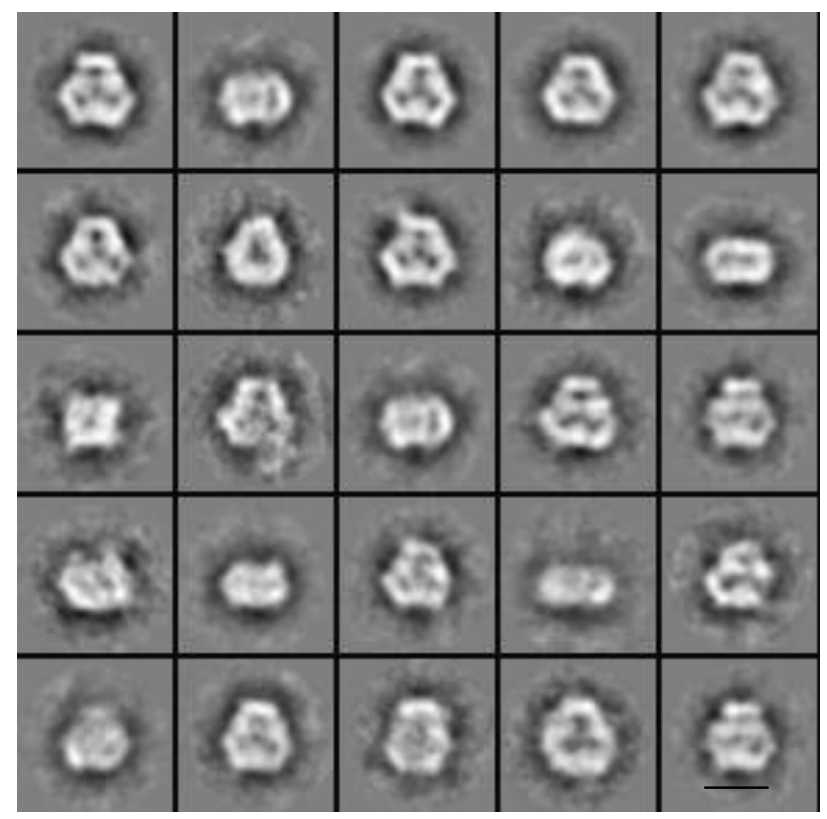

Fig. 1 Class sums of iniA particles. All scale bars correspond to $10 \mathrm{~nm}$.

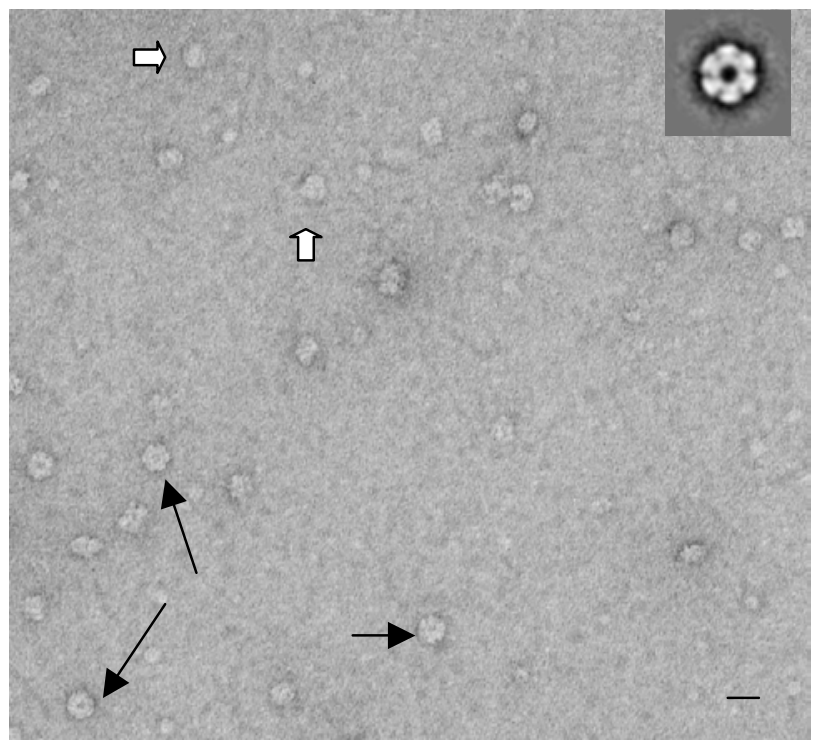

Fig. 3 Triangular(凹) and hexameric ring forms $(\rightarrow)$ of IniA.
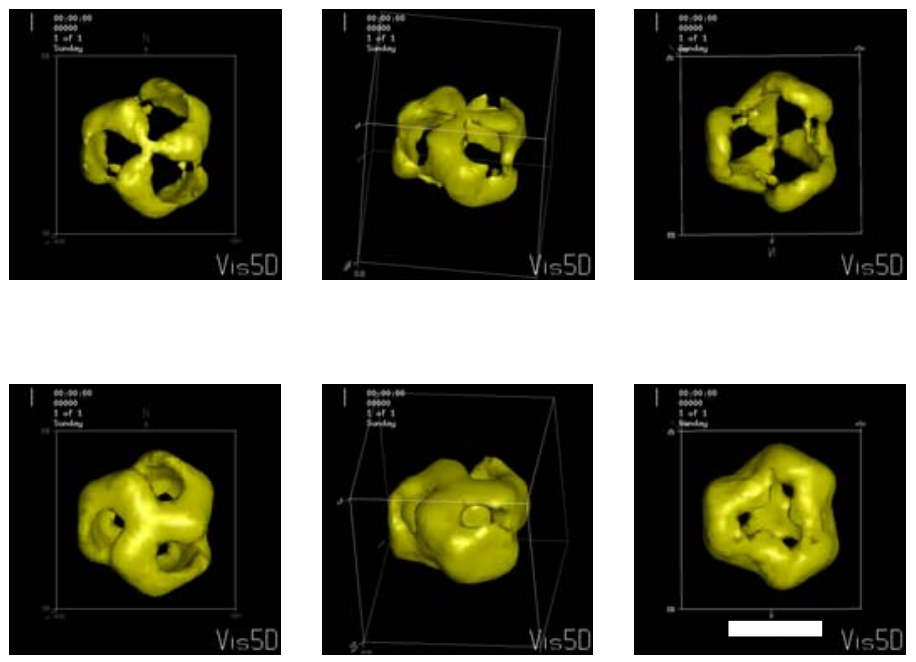

Fig. 2 3-D reconstructions using $210 \mathrm{kDa}$ (top row) and $438 \mathrm{kDa}$ (bottom row) thresholds.

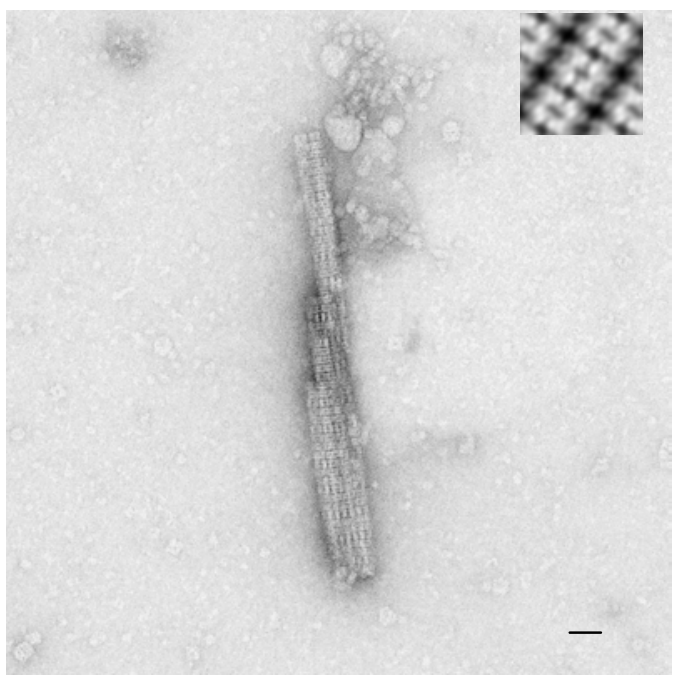

Fig. 4 Negatively stained crystal of IniA. Inset: Projection map calculated in $\mathrm{p} 2$. 